\title{
Total Hip Replacement: Exchange of a Loose Perforated Press-Fit Cup with a Porous-Coated Press-Fit Cup in a Dog
}

\author{
Stephanie M.M. Colthurst ${ }^{1} \quad$ James O. Simcock ${ }^{1} \quad$ Ricky G. Cashmore $^{1}$ \\ ${ }^{1}$ Southpaws Specialty Surgery for Animals, Moorabbin, Victoria, \\ Australia \\ Address for correspondence Stephanie Colthurst, BVSc, MANZCVS, \\ Southpaws Specialty Surgery for Animals, 3 Roper Street, Moorabbin, \\ VIC 3189, Australia (e-mail: stephanie.colthurst@gmail.com). \\ VCOT Open 2020;3:e129-e133.
}

\begin{abstract}
Keywords

- dog

- revision surgery

- cup exchange

- cementless

- total hip replacement

Objective The aim of this study was to report the successful revision of a loose perforated press-fit cup by exchange with a porous-coated press-fit cup while maintaining the original locking stem and head-neck unit.

Methods Cup revision was performed in a dog with a loose acetabular cup that had undergone total hip replacement with a perforated press-fit cup and locking stem 29 months previously. The original locking stem was well integrated. A single session cup exchange was performed by implantation of a 28 -mm porous-coated press-fit cup with $16 \mathrm{~mm}$ internal diameter to allow the original locking stem and head-neck unit to be preserved.

Results Revision total hip arthroplasty by cup exchange resulted in excellent functional outcome with long-term follow-up 18 months postoperatively. There were no intraoperative or postoperative complications.

Conclusion A loose perforated press-fit cup was successfully revised with a porous press-fit cup while allowing preservation of the initial locking stem and head-neck unit.
\end{abstract}

\section{Introduction}

Since the first report of canine total hip replacement (THR) by Gorman in 1957, improvements in implant design and surgical technique have led to its success as a treatment for various debilitating conditions of the coxofemoral joint in dogs. ${ }^{1-5}$ Implant systems are broadly divided into cemented and cementless systems. At present, commercially available cementless systems include the Kyon Zurich Cementless (ZTHR) (Kyon Inc., Zurich, Switzerland), BioMedtrix Biologic Fixation (BFX) (BioMedtrix, Boonton, New Jersey, United States) and the Helica Canine Cementless Hip System (Innoplant Veterinary, Hannover, Germany) systems. With all cementless systems, long-term stability requires osseointegration of patient bone into and/or onto the implant surface. ${ }^{6}$ The method of achieving initial short-term stability varies with the implant system used. The Z-THR system involves locking screws for immediate fixation of the stem and an

received

November 5, 2019

DOI https://doi.org/

accepted after revision

August 14, 2020 acetabular cup that is press-fit for immediate stability. ${ }^{7}$ Although first- and second-generation cups involved a central screw, it was not widely used given its use does not compensate for inaccurate press-fit. ${ }^{8,9}$ Newer generation cups involve a double-shell design with no central screw. Both the stem and cup have rough surfaces for ongrowth and the cup is additionally perforated with multiple small holes for ingrowth. Newest generation implants are also hydroxyapatite-coated to enhance osseointegration. The BFX system comprises of both a press-fit cup and stem with a porous surface created by electron beam melting three-dimensional printing into which bone ingrowth occurs for osseointegration. ${ }^{10}$ The Helica Canine Cementless Hip System involves positive profile self-tapping screw-in acetabular (Innoplant Screw Cup) and femoral (Helica TPS) components, both with rough surfaces for osseointegration. ${ }^{11,12}$

Reported major complication rates for canine cementless THR range from 15 to $28 \%$ for Z-THR, ${ }^{7,8,13,14} 11$ to $25 \%$ for
License terms

Stuttgart · New York

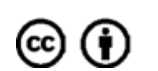


BFX $^{14-16}$ and 17 to $34.5 \%$ for Helica cementless. ${ }^{11,14,17,18}$ These include coxofemoral luxation, femoral fracture, implant loosening, implant fracture and infection. In the largest report of long-term outcomes of Z-THR, cup-associated complications of loosening, fracture and polyethylene wear were collectively the most common with an incidence of $6 \%$ overall. ${ }^{13}$ Treatment options for a loose acetabular cup include revision or explantation. Revision has been reported by impaction of a larger cementless cup ${ }^{8,19}$ or by use of a specifically designed Z-THR revision cup. ${ }^{20}$ This report describes the successful revision of a loose Z-THR perforated press-fit cup by exchange with a BFX porous-coated press-fit cup while maintaining the original Z-THR locking stem and head-neck unit, which has not been previously reported.

\section{Case Report}

A 43-month-old, $33 \mathrm{~kg}$, female spayed Labrador Retriever was presented for moderately severe left pelvic limb lameness. A left Z-THR had been performed 29 months previously for hip dysplasia associated osteoarthritis using a small stem, short neck and $23.5 \mathrm{~mm}$ cup. Radiographs 6 months following initial surgery suggested osseointegration of implants based on the absence of periprosthetic radiolucent lines and lack of implant migration or other concerns. The dog did clinically well until a mild weightbearing lameness developed 12 months after surgery with mild pain on hip extension. Radiographs at this time showed subtle loss of bone density around the acetabular component. Lameness resolved with activity restriction and non-steroidal anti-inflammatories. The dog was not represented for further investigation until 29 months after initial surgery.

On physical examination, there was an intermittent nonweight-bearing left pelvic limb lameness with pain on extension and abduction of the hip and marked left pelvic limb muscle atrophy. The dog was normothermic $\left(38.8^{\circ} \mathrm{C}\right)$ and otherwise well on general examination. Radiographs revealed a diffuse radiolucent line around the acetabular cup, particularly in zones I and III as described by DeYoung and colleagues. ${ }^{21}$ There was marked acetabular bone sclerosis with medial acetabular wall thickening. Movement of the cup was demonstrated on serial radiographs with the limb placed in various positions ( $\mathbf{- F i g . 1}$ ). The femoral component appeared well-integrated with no apparent implant loosening or migration.

\section{Revision Surgery}

Revision was performed 30 months following index surgery. Anaesthesia was routine with acepromazine and methadone premedication, alfaxalone induction and maintained with inhalational isoflurane. Lignocaine epidural was administered preoperatively and the dog received a fentanyl continuous rate infusion during surgery. Cefazolin $(22 \mathrm{mg} / \mathrm{kg}$ intravenously) was administered 30 minutes prior to surgery and every 90 minutes thereafter during surgery.

A standard craniolateral approach to the left hip joint was made. There was visible instability of the acetabular cup. The stem was seated securely with no evidence of gross instability. There was no gross evidence of infection, and a microbial culture and sensitivity sample was collected for completeness. The hip was luxated and the prosthetic head-neck unit was removed by externally rotating the femur and using a BFX impactor to carefully separate it from the stem. The removed head-neck unit was preserved in cefazolin-infused saline solution. The loose cup was easily removed and the acetabulum was prepared using BioMedtrix reamers of incremental increase in size beginning with a $21 \mathrm{~mm}$ starter reamer and ending with a $28 \mathrm{~mm}$ finishing reamer. The acetabular bone was noted to be extremely hard during reaming consistent with preoperatively documented sclerosis. A $28 \mathrm{~mm}$ BFX acetabular cup with an internal diameter of $16 \mathrm{~mm}$ (BioMedtrix, Boonton, New Jersey, United States) was seated with appropriate closure, inclination and version. The $16 \mathrm{~mm}$ diameter Z-THR head-neck unit was replaced and subjectively provided appropriate soft tissue tension. The hip was reduced and range of motion and implant stability was confirmed with manual tests. The site was lavaged copiously with cefazolin-infused saline (as is standard for all THRs performed at this practice) and a gentamicin-impregnated collagen sponge (Collatamp G, Syntacoll, Saal, Germany) was placed into the joint before routine closure. Postoperative radiographs showed good cup alignment (37 degrees angle of lateral opening, 5 degrees retroversion and 26 degrees inclination) with moderate $(3.8 \mathrm{~mm})$ polar gap. Recovery from anaesthesia was uneventful.

The dog was weight-bearing with moderate lameness when discharged 2 days postoperatively with meloxicam $(0.1 \mathrm{mg} / \mathrm{kg}$ orally once a day) for 2 weeks, codeine $(0.5$ $\mathrm{mg} / \mathrm{kg}$ orally twice a day [BID]) for 1 week, amoxicillinclavulanic acid $(23 \mathrm{mg} / \mathrm{kg}$ orally BID) for 2 weeks and acepromazine sedation $(0.75 \mathrm{mg} / \mathrm{kg}$ orally BID) for 4 weeks. Activity was restricted for 8 weeks. Leash walks and underwater treadmill therapy were introduced at 2 weeks after surgery and incrementally increased in duration throughout recovery.

At 9 days following surgery, the dog was ambulatory without lameness. Culture results returned negative and owners were instructed to continue the amoxicillin-clavulanic acid until the 14-day course was complete as was routine at the time at the practice where surgery was performed. At 2 months following surgery, the dog was clinically well. Radiographs revealed marked reduction in polar gap depth $(0.8 \mathrm{~mm})$ compared with immediate postoperative radiographs (-Fig. 2). At 6 months following surgery, there was excellent functional outcome with the dog running up to $3 \mathrm{~km}$ a day with the owner. Left pelvic limb musculature was now appreciably greater than the right pelvic limb which was also affected by coxofemoral osteoarthritis. Radiographs at this time revealed polar gap was no longer visible (-Fig. 2). There was no evidence of implant migration or other concerns and it was concluded that osseointegration had occurred. At 18 months following revision surgery, by telephone consultation, owners reported continued excellent function with no recurrence of lameness. 


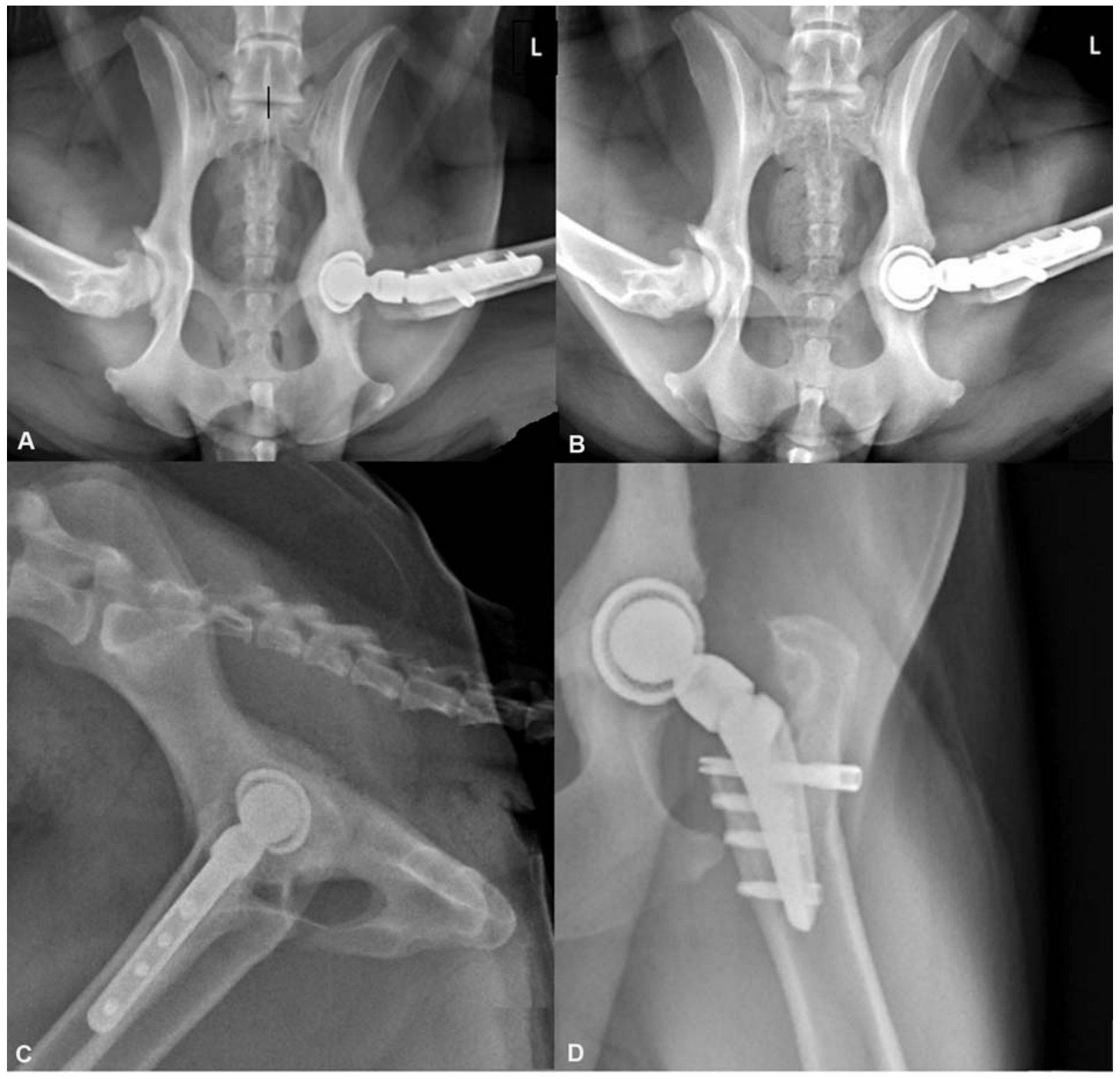

Fig. 1 (A-D) Radiographs 29 months following initial Z-THR. Parts (A and B) demonstrate gross movement of the acetabular cup is evident through serial projections with the limb in different positions. Panels $C$ and $D$ show no evidence of femoral implant loosening or migration.

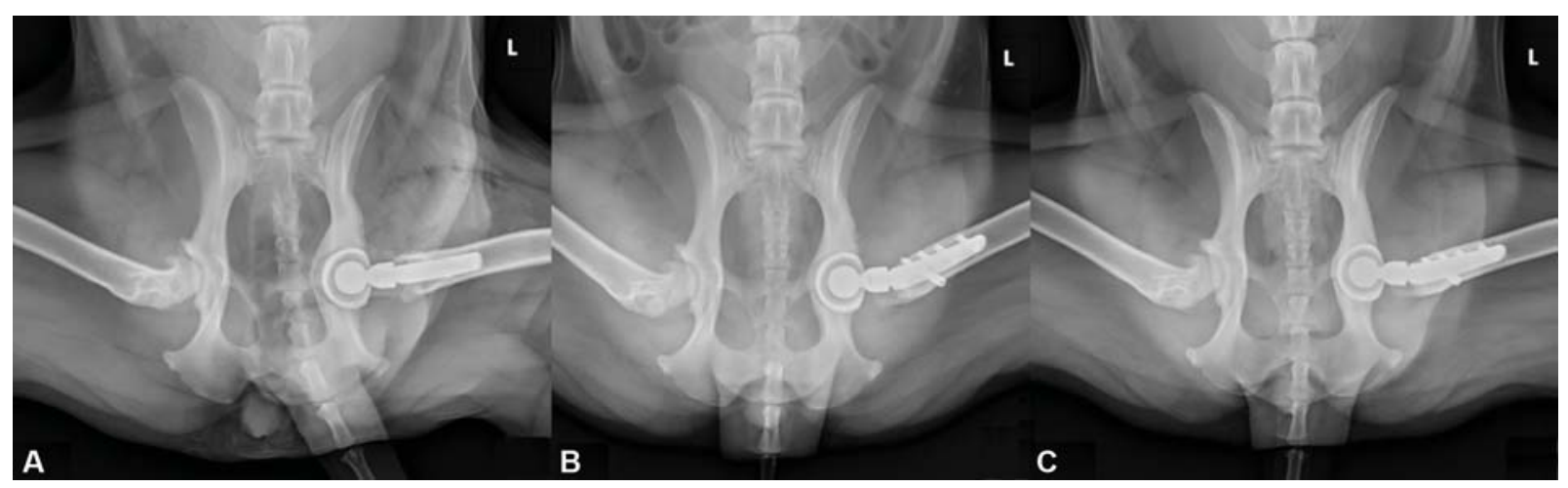

Fig. 2 (A) Immediate postoperative radiographs demonstrating moderate polar gap. (B) Eight-week postoperative radiographs demonstrating marked reduction in polar gap depth. (C) Twenty-four-week postoperative radiographs demonstrating gap fill. 


\section{Discussion}

To the author's knowledge, this is the first report of Z-THR to $B F X$ cup exchange. Revision was required due to marked pain and dysfunction secondary to a loose Z-THR cup. It cannot be confirmed whether the acetabular cup was initially stabilized by bone ingrowth that later failed, or if osseointegration never occurred and fibrous interface developed from onset.

In a long-term study of Z-THR, most cup-related complications, specifically polyethylene wear and cup fracture, involved the smaller diameter $(21.5$ and $23.5 \mathrm{~mm})$ cups with associated thinner liners, and were more commonly used in juvenile ( $\leq 11$ months old) dogs. It was speculated that cups were undersized for final adult size leading to premature wear, and that increase in body condition score over time contributed to failure. ${ }^{13}$ In the present case, index surgery was performed at 13 months of age. The $23.5 \mathrm{~mm}$ acetabular cup initially placed may have been relatively undersized leading to premature wear. A $26.5 \mathrm{~mm}$ cup may have been more appropriate and could also have been considered a revision option in this case. Body condition score was not consistently documented in this case; however, bodyweight in the first 6 months of index surgery was 27.3 to $28.0 \mathrm{~kg}$ compared with 32.8 to $33.0 \mathrm{~kg}$ during the 6 months surrounding revision surgery. Increased body condition is suspected but cannot be confirmed, which may have also contributed to failure of the original Z-THR cup in this case.

Loss of acetabular bone stock and poor bone quality are concerns when implanting new cementless cups. Achieving immediate stability and minimizing micromotion are paramount for osseointegration to occur. ${ }^{22}$ In the present case, reaming was continued to the medial wall to allow deeper seating of the BFX cup. We suspect that the remodelled sclerotic acetabular bed did not allow for complete cup impaction which left a moderate polar gap evident on immediate postoperative radiographs (see - Fig. 2). Polar gap, a radiolucent zone between the acetabular cup-bone interface, occurs from absence of implant-bone contact. Polar gap may occur with poor acetabular bone preparation, abnormal acetabular bone quality (as is suspected in this case), insufficient impaction, premature locking of the implant (when impacting the cup out of line to bed preparation) and cup stiffness. Liska and colleagues identified polar gap in the majority (68\%) of immediate postoperative radiographs following 200 BFX hip replacements, all of which resolved without complication leading the authors to conclude that polar gaps do not warrant clinical intervention. ${ }^{23}$ In that study, gaps ranged from $<1$ to $4 \mathrm{~mm}$ with gap fill complete by 5 weeks postoperatively in all cases. This is in contrast to the present case where a thin polar gap remained present at 8 weeks. This is suspected due to the significant initial gap depth $(3.8 \mathrm{~mm})$ and very sclerotic bone (cancellous poor) present in this case. At 24 weeks following surgery, polar gap was no longer visible in the present case.

Normally Z-THR and BFX systems are incompatible due to differences in the internal diameter of the cup and diameter of the corresponding femoral heads. Standard BFX cups have a $12,13,14,17$ or $22 \mathrm{~mm}$ internal diameter with corresponding head sizes, whereas the internal diameter of Z-THR cups and their corresponding heads are available in 16 and $19 \mathrm{~mm}$. Production of a BFX cup with an internal diameter of $16 \mathrm{~mm}$ allowed the Z-THR femoral components to be maintained in this case. Several other options could have been considered for revision including use of a larger Z-THR cup, use of the specifically designed Z-THR revision cup, use of a Helica cup or use of a neck adaptor produced by Kyon to allow use of a standard BFX cup with $17 \mathrm{~mm}$ internal diameter and normal BFX head. ${ }^{20,24}$ Recently, use of a three-dimensional printed custom acetabular prosthesis has been reported in a dog with significant bone loss after femoral head and neck excision which could also have been considered. ${ }^{25}$ Finally, removal of the Z-THR stem for a BFX stem exchange was yet another possibility; however, it was considered unnecessary given the Z-THR stem was assessed to be stable. Removal of a well-integrated stem could risk femoral fracture and significant bone loss, complicating the insertion and stability of a new stem. In the present case, the ultimate decision to use a BFX cup was based on the low reported complications relating to the BFX acetabular component and surgeonfamiliarity with this system.

Reported cup complication rates are 3 to $6 \%$ for Z-THR $7,8,13$ compared with $1 \%$ for BFX cups. ${ }^{16}$ Stem-related complications are rare with the Z-THR system with reported rates of 1.7 to $4 \% 7,8,13$ compared with 4.4 to $24 \%$ for the BFX stem. ${ }^{15,16}$ The low complication rate of the BFX cup and ZTHR stem has led some to propose this hybrid combination as the ideal choice of implants for THR in dogs. ${ }^{24}$ Although two cases of successful exchange of loose BFX press-fit stems with Z-THR stems while maintaining the BFX cup have been reported, ${ }^{24}$ hybrid BFX cup and Z-THR stem at index surgery (as opposed to revision) THR are yet to be reported.

Based on hypothesized lower complication rate for the ZTHR stem component and lower luxation rates for the Helica Canine Cementless Hip system, Bayer and colleagues retrospectively reviewed the complications and long-term outcome in 16 dogs undergoing cementless hybrid THR using an Innoplant Screw Cup coupled with a Z-THR head and stem. There was full restoration of function in 15 dogs and acceptable restoration of function in the remaining dog. Three dogs (19\%) experienced major complications including dorsal luxation, aseptic cup loosening and aseptic stem loosening and subsidence. Given the comparable complication rate to single implant THR systems, while clinically feasible, the authors concluded there was no greater benefit using the hybrid system in primary THR surgery. ${ }^{26}$

In the current case, the original Z-THR head-neck unit was maintained given it was grossly undamaged and because it was considered the appropriate length at time of reduction. Medium, long and extra-long Z-THR head-neck units were available at the time of revision surgery should reduction have been too loose. In humans, both cup exchange and liner exchange are always combined with head exchange. ${ }^{27}$ The authors acknowledge that although no gross damage was visible, any microscopic damage to the surface of the head could lead to accelerated wear of the newly placed acetabular 
cup. Ultimately, it was intraoperatively assessed that the use of the original head-neck unit was appropriate, the simplest method, and with no further added expense.

Although this is a single case report, results suggest revision of a loose Z-THR acetabular cup with a BFX cup is a feasible technique with excellent short- and medium-term outcomes achieved.

\section{Authors' Contributions}

R.G.C. and J.O.S. planned and performed the described revision surgery. S.M.M.C. prepared the manuscript with support from R.G.C. and J.O.S.

\section{Conflicts of Interest}

None declared.

\section{References}

1 Gorman HA. A new prosthetic hip joint; experiences in its use in the dog, and its probable application to man. Mil Med 1957;121 (02):91-93

2 Olmstead ML, Hohn RB, Turner TM. A five-year study of 221 total hip replacements in the dog.J Am Vet Med Assoc 1983;183(02):191-194

3 Liska WD. Cemented total hip replacement: experience in the USA with the BioMedtrix prosthesis. In: Proceedings of the 2004 European Society of Veterinary Orthopedics and Traumatology, Pre-Congress - Total Hip Replacement Seminar, 2004Munich, Germany

4 Iwata D, Broun HC, Black AP, Preston CA, Anderson GI. Total hip arthroplasty outcomes assessment using functional and radiographic scores to compare canine systems. Vet Comp Orthop Traumatol 2008;21(03):221-230

5 Skurla CT, Egger EL, Schwarz PD, James SP. Owner assessment of the outcome of total hip arthroplasty in dogs. J Am Vet Med Assoc 2000;217(07):1010-1012

6 Khanuja HS, Vakil JJ, Goddard MS, Mont MA. Cementless femoral fixation in total hip arthroplasty. J Bone Joint Surg Am 2011;93 (05):500-509

7 Hummel DW, Lanz OI, Werre SR. Complications of cementless total hip replacement. A retrospective study of 163 cases. Vet Comp Orthop Traumatol 2010;23(06):424-432

8 Guerrero TG, Montavon PM. Zurich cementless total hip replacement: retrospective evaluation of 2 nd generation implants in 60 dogs. Vet Surg 2009;38(01):70-80

9 Hanson SP, Peck JN, Berry CR, Graham J, Stevens G. Radiographic evaluation of the Zurich cementless total hip acetabular component. Vet Surg 2006;35(06):550-558

10 Schiller TD. BioMedtrix total hip replacement systems: an overview. Vet Clin North Am Small Anim Pract 2017;47(04):899-916

11 Hach V, Delfs G. Initial experience with a newly developed cementless hip endoprosthesis. Vet Comp Orthop Traumatol 2009;22(02):153-158
12 Harper TAM. INNOPLANT total hip replacement system. Vet Clin North Am Small Anim Pract 2017;47(04):935-944

13 Vezzoni L, Vezzoni A, Boudrieau RJ. Long-term outcome of Zürich cementless total hip arthroplasty in 439 cases. Vet Surg 2015;44 (08):921-929

14 Henderson ER, Wills A, Torrington AM, et al. Evaluation of variables influencing success and complication rates in canine total hip replacement: results from the British Veterinary Orthopaedic Association Canine Hip Registry (collation of data: 20102012). Vet Rec 2017;181(01):18

15 Roe S, Marcellin-Little D, Lascelles D. Short-term outcome of uncemented THR. In: Proceedings of the 2010 American College of Veterinary Surgeons, Veterinary Symposium; 2010Germantown, Maryland

16 Kidd SW, Preston CA, Moore GE. Complications of porous-coated press-fit cementless total hip replacement in dogs. Vet Comp Orthop Traumatol 2016;29(05):402-408

17 Agnello KA, Cimino Brown D, Aoki K, Franklin S, Hayashi K. Risk factors for loosening of cementless threaded femoral implants in canine total hip arthroplasty. Vet Comp Orthop Traumatol 2015; 28(01):48-53

18 Denny HR, Linnell M, Maddox TW, Comerford EJ. Canine total hip replacement using a cementless threaded cup and stem: a review of 55 cases. J Small Anim Pract 2018;59(06): 350-356

19 Nesser VE, Kowaleski MP, Boudrieau RJ. Severe polyethylene wear requiring revision total hip arthroplasty in three dogs. Vet Surg 2016;45(05):664-671

20 Vezzoni L, Montinaro V, Vezzoni A. Use of a revision cup for treatment of Zurich cementless acetabular cup loosening. Surgical technique and clinical application in 31 cases. Vet Comp Orthop Traumatol 2013;26(05):408-415

21 DeYoung DJ, Schiller RA, DeYoung BA. Radiographic assessment of a canine uncemented porous-coated anatomic total hip prosthesis. Vet Surg 1993;22(06):473-481

22 El-Warrak AO, Olmstead ML, von Rechenberg B, Auer JA. A review of aseptic loosening in total hip arthroplasty. Vet Comp Orthop Traumatol 2001;14(03):115-124

23 Liska WD, Israel SK, Poteet BA. Polar gap after cementless total hip replacement in dogs. Vet Surg 2019;48(03):321-335

24 Vezzoni L, Bazzo S, Vezzoni A. Revision of a BFX total hip replacement stem using a Kyon stem and a head adaptor in two dogs. Vet Comp Orthop Traumatol 2017;30(01):81-87

25 Castelli E, Schmierer PA, Pozzi A. Custom acetabular prosthesis for total hip replacement: a case report in a dog with acetabular bone loss after femoral head and neck ostectomy. Vet Surg 2019;48 (08):1520-1529

26 Bayer K, Matiasovic M, Steger H, Böttcher P. Complications and long-term outcome in 16 canine cementless hybrid hip arthroplasties. Vet Comp Orthop Traumatol 2019;32(01): 73-78

27 Walmsley DW, Waddell JP, Schemitsch EH. Isolated cup and liner exchange in revision hip arthroplasty. J Am Acad Orthop Surg 2017;25(04):288-296 